\title{
Follow-Up of Incidental High-Risk Pulmonary Nodules on Computed Tomography Pulmonary Angiography at Care Transitions
}

\author{
Janice L Kwan, MD, MPH ${ }^{1,2 *}$; Darya Yermak, MD²; Lezlie Markell, MB, BCh BAO²; Narinder S Paul, MD³; \\ Kaveh G Shojania, MD²,4 Peter Cram, MD, MBA ${ }^{1,2}$
}

${ }^{1}$ Division of General Internal Medicine, Mount Sinai Hospital, Toronto, Ontario, Canada; ${ }^{2}$ Department of Medicine, University of Toronto, Toronto, Ontario, Canada; ${ }^{3}$ Department of Medical Imaging, Western University, London, Ontario, Canada; ${ }^{4}$ Division of General Internal Medicine, Sunnybrook Health Sciences Centre, Toronto, Ontario, Canada.

BACKGROUND: Computed tomography pulmonary angiography (CTPA) detects incidental findings that require follow-up. In just over $50 \%$ of cases, those incidental findings are pulmonary nodules. Fleischner guidelines recommend that patients with nodules that have a high risk of malignancy should undergo CT followup within 3-12 months.

OBJECTIVE: We examined the proportion of patients with pulmonary nodules requiring follow up who received repeat imaging within six weeks of the time frame recommended by the radiologist.

DESIGN: This retrospective cohort study included all patients who underwent CTPA in the emergency department and inpatient settings at three teaching hospitals in Toronto, Canada between September 1, 2014, and August 31, 2015. Natural language processing software was applied to a linked radiology information system to identify all CTPAs that contained pulmonary nodules. Using manual review and prespecified exclusion criteria, we generated a cohort with possible new lung malignancy eligible for follow-up imaging; then we reviewed available health records to determine whether follow-up had occurred.

RESULTS: Of the 1,910 CTPAs performed over the study period, 674 (35.3\%) contained pulmonary nodules. Of the 259 patients with new nodules eligible for follow-up imaging, 65 received an explicit suggestion for follow-up by radiology (25.1\%). Of these 65 patients, 35 (53.8\%) did not receive repeat imaging within the recommended time frame. Explicit mention that follow-up was required in the discharge summary $(P=.03)$, attending an outpatient follow-up visit $(P<.001)$, and younger age $(P=.03)$ were associated with receiving timely follow-up imaging.

CONCLUSIONS: Over $50 \%$ of patients with new highrisk pulmonary nodules detected incidentally on CTPA did not receive timely follow-up imaging. Journal of Hospital Medicine 2019; 14 349-352. Published online first February 20, 2019. (C) 2019 Society of Hospital Medicine omputed tomography pulmonary angiography (CTPA) is often used in the evaluation of suspected pulmonary embolism (PE). The detection of incidental findings that require follow-up is common; in just over $50 \%$ of cases, those incidental findings are pulmonary nodules. ${ }^{1}$ Although the majority of these nodules are benign, Fleischner Society guidelines ${ }^{2}$ recommend that patients with nodules at high risk for malignancy should undergo follow-up CT imaging within 3-12 months, with patients who smoke and have large nodules requiring closer follow up.

The failure to follow-up on abnormal test results is known to contribute to diagnostic error and can lead to patient harm. ${ }^{3}$ We sought to determine the proportion of high-risk pulmonary nodules on CTPA which did not undergo the recommended follow-up imaging.

\footnotetext{
*Corresponding Author: Janice L. Kwan; E-mail: janice.kwan@utoronto.ca; Telephone: (416) 586-4800; Twitter: @KwanJanice
}

Received: July 19, 2018; Revised: October 29, 2018; Accepted: November 18, 2018

๑ 2019 Society of Hospital Medicine DOI 10.12788/jhm.3128

\section{METHODS}

\section{Study Setting and Design}

This retrospective cohort study included all patients who underwent CTPA in the emergency department (ED) and inpatient settings at three academic health centers (Mount Sinai Hospital, Toronto General Hospital, and Toronto Western Hospital) in Toronto, Canada between September 1, 2014, and August 31, 2015.

We examined the proportion of patients with pulmonary nodules requiring follow up who received repeat CT imaging within six weeks of the time frame recommended by the radiologist. Since we were interested in measuring the rate of an important test result that is missed (rather than accuracy of the test itself), we defined "requiring follow up" as the inclusion of explicit recommendations for follow up in the radiology report.

Montage (Philadelphia, Pennsylvania), a natural language processing software, was applied to a linked radiology information system (RIS) to identify all CTPAs that contained pulmonary nodules. We conducted manual chart review to confirm software accuracy. We initially searched the RIS for all CTPAs that were completed within the study period, resulting in the identification of 1932 imaging studies. Following a review of 


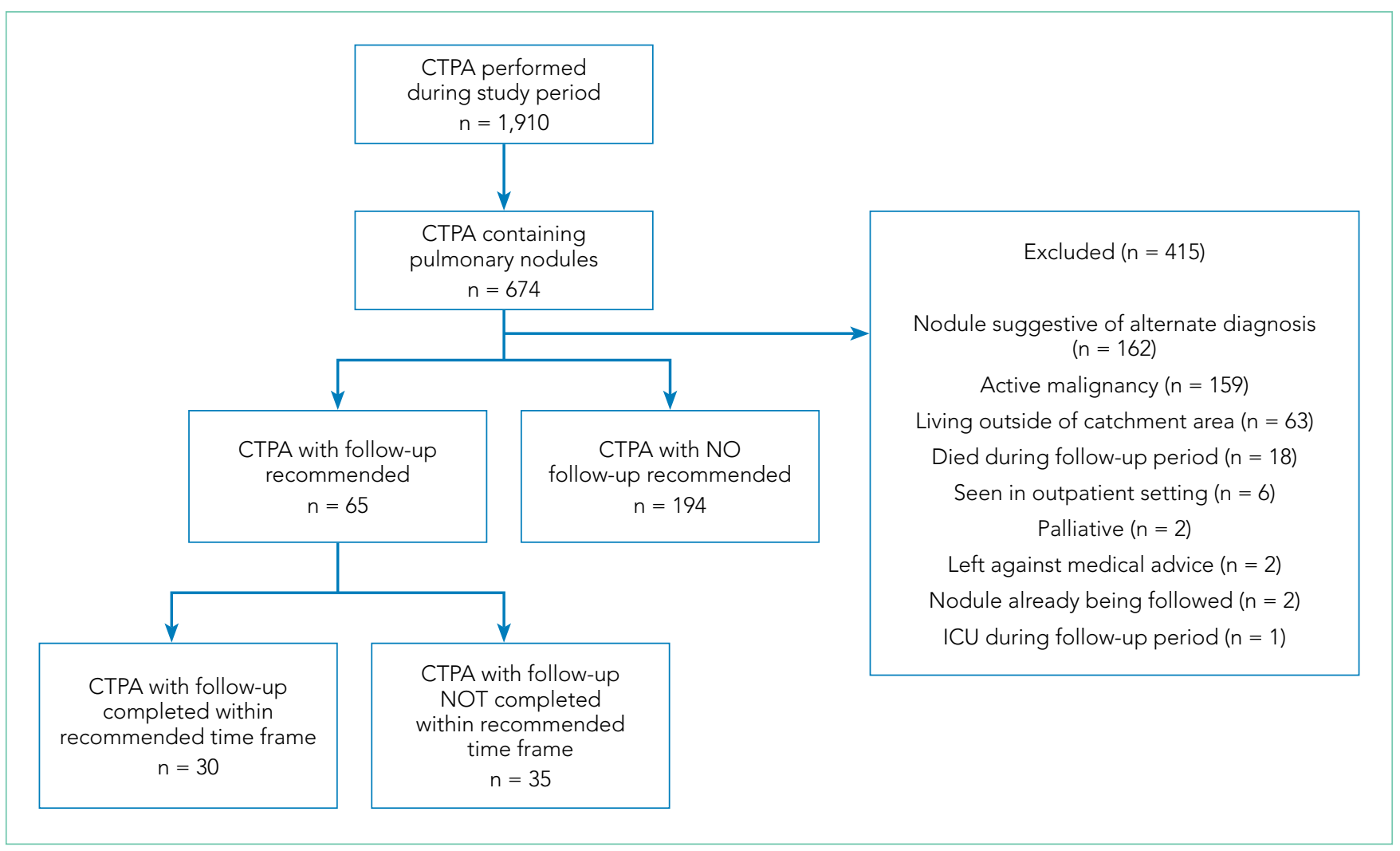

FIG. Flowchart of Computed Tomography Pulmonary Angiography (CTPA) Scans Included in the Study Cohort.

these 1,932 studies, we excluded 22 as they were not CTPAs. We then applied the search term, "nodule-" to 1,910 confirmed CTPAs, resulting in the identification of 836 imaging studies. Following a review of these 836 studies, we excluded 10 as they were duplicate studies. We also excluded 152 studies where the term "nodule" did not identify a pulmonary nodule but instead referred to a radiologist reporting the absence of pulmonary nodules (eg "there were no pulmonary nodules found") or the presence of non-lung nodules (eg thyroid nodules). This resulted in the identification of 674 CTPAs containing pulmonary nodules (Figure 1).

Thereafter, we generated a cohort with possible new lung malignancy eligible for follow-up imaging by reviewing available health records and applying the following prespecified exclusion criteria: (1) patients who died, (2) left against medical advice, (3) were critically ill during the follow-up period, (4) lived outside the hospital catchment area (Greater Toronto Area), (5) were seen in the outpatient setting, (6) identified as palliative, (7) had an active malignancy, (8) had nodules that were already being followed, or (9) had nodules with characteristics suggestive of alternate diagnoses to lung malignancy (such as infection or inflammation) with no follow up recommended as reported by the radiologist. For patients with multiple CTPAs, we included only the first study. For each eligible patient, we determined whether follow-up imaging was completed by manually reviewing the linked RIS. We reviewed available health records to determine whether the pulmonary nodule findings had been discussed with the patient and whether the patient had attended an outpatient follow-up visit. In patients for whom recommended follow-up imaging was not confirmed, we notified the ordering physician by e-mail.

Each radiology department followed the same protocol adherent to the 2005 Fleischner guidelines for identifying nodules requiring follow up. ${ }^{2}$ Virtually all CTPAs at the three study institutions are read and reported within 72 hours. The ordering physician is sometimes called at the discretion of the reading radiologist when the findings are judged to be urgent and time-sensitive in nature. For example, the ordering physician may be contacted if a CTPA is positive for segmental PE but is not typically called for incidental pulmonary nodules. It is not common practice for ordering physicians to be notified of incidental findings above and beyond the radiology report. Primary care physicians are not typically copied on radiology reports and usually do not use the same electronic health record.

\section{Statistical Analysis}

We calculated simple descriptive statistics for all results. Mean values were compared using two-tailed t-tests, categorical groups using chi-square tests, and median values using Mann-Whitney U tests. We performed all analyses using Microsoft Excel version 16.14.1 (Redmond, Washington).

\section{Ethics Approval}

This study was approved by each institution's research ethics board. 
TABLE. Characteristics of Patients with High-Risk Pulmonary Nodules Noted on Computed Tomography Pulmonary Angiography Requiring Follow Up.

\begin{tabular}{|c|c|c|c|c|}
\hline \multirow[b]{2}{*}{ Characteristic } & \multicolumn{4}{|c|}{ No. $(\%)$} \\
\hline & $\begin{array}{c}\text { All } \\
(n=65)\end{array}$ & $\begin{array}{l}\text { Follow Up Completed } \\
\text { Within the Recommended Time Frame } \\
\qquad(\mathrm{n}=30)\end{array}$ & $\begin{array}{l}\text { Follow Up NOT Completed } \\
\text { Within the Recommended Time Frame } \\
\qquad(\mathrm{n}=35)\end{array}$ & $P$ Value \\
\hline \multicolumn{5}{|l|}{ Sociodemographics } \\
\hline Mean age (Standard Deviation), y & $67.2(15.2)$ & $62.8(15.7)$ & $71.0(14.0)$ & .03 \\
\hline Women & $33(51)$ & $14(47)$ & $19(54)$ & .29 \\
\hline \multicolumn{5}{|l|}{ Lung Cancer Risk Factor } \\
\hline \multicolumn{5}{|l|}{ Never Smoked } \\
\hline Yes & $11(17)$ & $6(20)$ & $5(14)$ & .42 \\
\hline No & $36(55)$ & $18(60)$ & $18(52)$ & \\
\hline Unknown & $18(28)$ & $6(20)$ & $12(34)$ & \\
\hline \multicolumn{5}{|l|}{ Chronic Obstructive Pulmonary Disease } \\
\hline Yes & $24(37)$ & $10(33)$ & $14(40)$ & .58 \\
\hline No & $41(63)$ & $20(67)$ & $21(60)$ & \\
\hline \multicolumn{5}{|l|}{ Imaging } \\
\hline \multicolumn{5}{|l|}{ Lung Nodule } \\
\hline Single & $12(19)$ & $5(17)$ & $7(20)$ & .73 \\
\hline Multiple & $53(81)$ & $25(83)$ & $28(80)$ & \\
\hline Median Recommended Follow-Up (Interquartile Range), months & $6(3-12)$ & $6(3-12)$ & $6(3-12)$ & .90 \\
\hline \multicolumn{5}{|l|}{ System } \\
\hline \multicolumn{5}{|l|}{ Hospital Department } \\
\hline Emergency department & $27(42)$ & $14(47)$ & $13(37)$ & .62 \\
\hline Inpatient & $38(58)$ & $16(53)$ & $22(63)$ & \\
\hline \multicolumn{5}{|l|}{ Follow-Up Instructions Included in the Discharge Summary } \\
\hline Included & $36(55)$ & $21(70)$ & $15(43)$ & .03 \\
\hline Not included & $29(45)$ & $9(30)$ & $20(57)$ & \\
\hline \multicolumn{5}{|l|}{ Attended Outpatient Follow-Up Visit } \\
\hline Yes & $18(28)$ & $16(53)$ & $2(6)$ & $<.001$ \\
\hline No & $47(72)$ & $14(47)$ & $33(94)$ & \\
\hline
\end{tabular}

\section{RESULTS}

Follow Up of Incidental High-Risk Pulmonary Nodules Of the 1910 CTPAs performed over the study period (Figure), 674 (35.3\%) contained pulmonary nodules. Of the 259 patients with new pulmonary nodules eligible for follow-up imaging, 194 (74.9\%) did not have an explicit suggestion for follow up by the radiologist. Ninety-five percent of radiologists (184 out of 194) provided an explanation for not recommending follow up in the radiology report; the two most common reasons were small nodule size (often described as "tiny") and no interval change compared with the prior imaging study. ${ }^{2}$ Of the 65 patients who did receive an explicit suggestion for follow up by radiology, 35 (53.8\%) did not receive repeat imaging within the recommended time frame, allowing for a six-week grace period. Of these 35 patients, 10 eventually went on to receive delayed repeat imaging. The median follow-up time for the 30 patients who received timely repeat imaging was four months (IQR 2-6 months); in contrast, the median follow-up time for the 10 patients who received delayed repeat imaging was seven months (IQR 6-8 months), $P=.01$.

Of the 65 patients for whom follow up was recommended, the medical record showed evidence that there was a discussion between the medical team and the patient regarding patient preference for or against follow up in $55.4 \%$ (36 out of 65) of the patients. Notably, all 36 patients showed interest in receiving follow up; no patient indicated a preference for no follow up.

Furthermore, of the 65 patients that had follow up recommended, two patients were eventually diagnosed with lung cancer (one via lung biopsy, the other via positron emission 
tomography imaging); both patients did not receive timely follow-up imaging. While we did not include nodule size as an exclusion criterion, not one of the 65 patients included in the final cohort had nodules larger than $3 \mathrm{~cm}$.

\section{Physician Notification}

In circumstances where we could not confirm that followed up had occurred, we notified the ordering physician by e-mail. Since 10 of the 35 patients who did not receive timely follow-up imaging went on to receive delayed repeat imaging, we notified 25 physicians. Of the 25 physicians that we e-mailed, 24 acknowledged receipt of the information. Of these 24 physicians, 14 reported conducting a detailed review of the chart, from which the following additional information was obtained: one patient expired, and five physicians notified the corresponding primary care physicians (two of whom were unaware of the nodule, and subsequently arranged further follow up with the patient).

\section{Characteristics Associated with Timely Follow Up}

Explicit mention that follow up was required in the discharge summary $(P=.03)$, attending an outpatient follow-up visit $(P<$ $.001)$, and younger age $(P=.03)$ were associated with receiving timely follow up; patient sex, smoking history, history of chronic obstructive pulmonary disease, lung nodule count, recommended follow-up time, and hospital department (defined as the discharging service) were not (Table).

\section{DISCUSSION}

In this multicenter cohort study, over $50 \%$ of patients with new high-risk pulmonary nodules detected incidentally on CTPA did not receive timely follow-up imaging. Including follow-up recommendations in the discharge summary, attending an outpatient follow-up visit, and younger age were associated with timely follow-up imaging.

Few studies have assessed the follow up of incidental nodules identified on CTPA. In a retrospective cohort study of ED patients in the United States, Blagev et al. found that only $29 \%$ received timely follow up. ${ }^{4}$ Our study contributes to the literature in several ways. First, our study included all hospitalized patients, not only those in the ED. Notably, most of our cohort were inpatients, a group of patients not previously described. Second, we examined factors associated with timely follow up, which may help to inform future quality improvement initiatives and interventions. Third, we included data from three different hospitals, which may improve generalization. Lastly, our study draws on contemporary Canadian data. Most of the studies investigating test result follow up have been conducted in the US, 5 and Europe, ${ }^{7}$ with few empirical studies describing this phenomenon within the Canadian healthcare setting. We believe that our work contributes to the existing evidence that missed test results occur across diverse healthcare systems and have yet to be solved. ${ }^{5-7}$

Our study had limitations. First, we defined follow up as repeat imaging and did not include office visits or biopsy in this definition. Second, we may have missed repeat imaging and outpatient follow-up visits that occurred outside the study hospitals. Although we were able to determine if repeat imaging and outpatient follow-up visits (eg, pulmonology or thoracic surgery clinics) had occurred within the study hospitals, we did not have access to follow-up encounters that occurred outside of the study hospitals (eg primary care clinics). We are unaware of any published regional data on the rate of outpatient follow up at the index facility following discharge. However, we know from provincial data of patients discharged from the ED with a new cardiac diagnosis that just under half are seen by a family physician, cardiologist, or internist within seven days, with just under $80 \%$ seen within 30 days. ${ }^{8}$ Third, although we attempted to capture patient preference for or against repeat imaging using chart review, the absence of documentation of patient preference did not confirm that a discussion regarding patient preferences had not occurred. Fourth, while we did exclude patients that had an active malignancy, we did not exclude patients who were younger than 35 years or were immunocompromised, which may have led to an overestimation of the percentage of patients who did not receive follow up.

Incidental findings detected on acute diagnostic tests requiring handoffs for chronic follow up are at risk of falling through the cracks. The inclusion of follow-up recommendations in discharge summaries has been shown to increase the likelihood of follow-up completion. ${ }^{9}$ Our study provides additional evidence of the urgent need for interventions aimed at closing the loop on test result follow up. ${ }^{5,6}$

Disclosures: None of the authors have any conflicts of interest to disclose in reference to this study.

Funding: JLK is supported by the Mount Sinai Hospital Department of Medicine Research Fund. PC is supported by a K24 award from the National Institute of Arthritis and Musculoskeletal and Skin Diseases (AR062133).

\section{References}

1. Hall WB, Truitt SG, Scheunemann LP, et al. The prevalence of clinically relevant incidental findings on chest computed tomographic angiograms ordered to diagnose pulmonary embolism. Arch Intern Med 2009;169(21):1961. doi: 10.1001/archinternmed.2009.360.

2. Macmahon H, Austin JHM, Gamsu G, et al. Guidelines for Management of Small Pulmonary Nodules Detected on CT Scans: A Statement from the Fleischner Society. Radiology 2005;237(2):395-400. doi: 10.1148/radiol.2372041887.

3. National Academies of Sciences, Engineering, and Medicine. Improving diagnosis in health care. Washington, DC. 2015.

4. Blagev DP, Lloyd JF, Conner K, et al. Follow-up of incidental pulmonary nodules and the radiology report. J Am Coll Radiol 2014;11(4):378-383. doi: 10.1016/j.jacr.2013.08.003.

5. Callen J, Georgiou A, Li J, Westbrook Jl. The safety implications of missed test results for hospitalized patients: a systematic review. BMJ Quality Safety 2011;20(2):194-199. doi: 10.1136/bmjqs.2010.044339.

6. Callen JL, Westbrook JI, Georgiou A, Li J. Failure to follow-up test results for ambulatory patients: a systematic review. J Gen Intern Med 2011;27(10):13341348. doi: 10.1007/s11606-011-1949-5

7. Litchfield I, Bentham L, Lilford R, Mcmanus RJ, Hill A, Greenfield S. Test result communication in primary care: a survey of current practice. BMJ Quality Safety 2015;24(11):691-699. doi: 10.1136/bmjqs-2014-003712.

8. Atzema $\mathrm{CL}, \mathrm{Yu} \mathrm{B}$, Ivers NM, et al. Predictors of obtaining follow-up care in the province of Ontario, Canada, following a new diagnosis of atrial fibrillation, heart failure, and hypertension in the emergency department. Cjem 2017;20(03):377-391. doi: 10.1017/cem.2017.371.

9. Moore C, McGinn T, Halm E. Tying up loose ends: Discharging patients with unresolved medical issues. Arch Intern Med 2007;167(12):1305-1311. doi: 10.1001/archinte.167.12.1305 